\title{
Acute Neck Cellulitis Revealing Cutaneous Non Hodgkinien B Cell Lymphomas: Case Report and Review of the Literature
}

\author{
Beghdad M*, Laachoubi M, Merzouki B, Oukessou Y, Rouadi S, Abada R, Roubal M and Mahtar M \\ Faculty of Medicine of Casablanca, Department of Otolaryngology, hospital 20 august Morocco
}

*Corresponding author: Beghdad M, Faculty of Medicine of Casablanca, Department of Otolaryngology, hospital 20 august Morocco.

\begin{abstract}
Cutaneous lymphomas are lymphocyte proliferations. from cutaneous lymphoid tissue, without nodal, medullary, or visceral location. Their clinical presentation is quite polymorphic, and cellulitis is one of the modes of revelation, particularly forehead and neck location. They have no portal of entry and are resistant to treatment. Diagnosis is based on histology, and biopsies must be taken if lymphoma is suspected. The treatment is radio and chemotherapy, and the evolution depends on the tumoral stage. We report a case of cervical cellulitis revealing a cutaneous lymphoma in a 43-year-old male patient. The diagnosis was proved by multiple biopsies, performed because there was no clinical improvement in spite of an aggressive and adequate antibiotherapy.
\end{abstract}

\section{Introduction}

Cutaneous lymphomas represent a well-defined nosological entity. It is a lymphocytic proliferation with a cutaneous starting point, without lymph node, visceral or medullary invasion six months after diagnosis [1]. Their clinical manifestations are very polymorphic, often misleading, ranging from a simple skin papule to a pattern of soft tissue cellulitis. The diagnosis is purely histological, and their classification is based on the criteria defined by the EORTC group (European Organisation for Research and Treatment of Cancer). which takes into account the clinic, the morphology, the immunophenotype and evolution [1,2].

\section{Case Report}

A 43-year-old patient with no history of disease. was presented to the emergency department with erythema, tenderness, local heat of the left laterocervical region evolving since 7 days in a context of fever and alteration of the general state. The clinical examination revealed a left laterocervical shielding, very inflammatory, sensitive, with zones of cutaneous necroses (Figure

1). Faced with this condition, we immediately began an antibiotic treatment by parenteral administration combining amoxicillin and aminoglycosides in high doses.

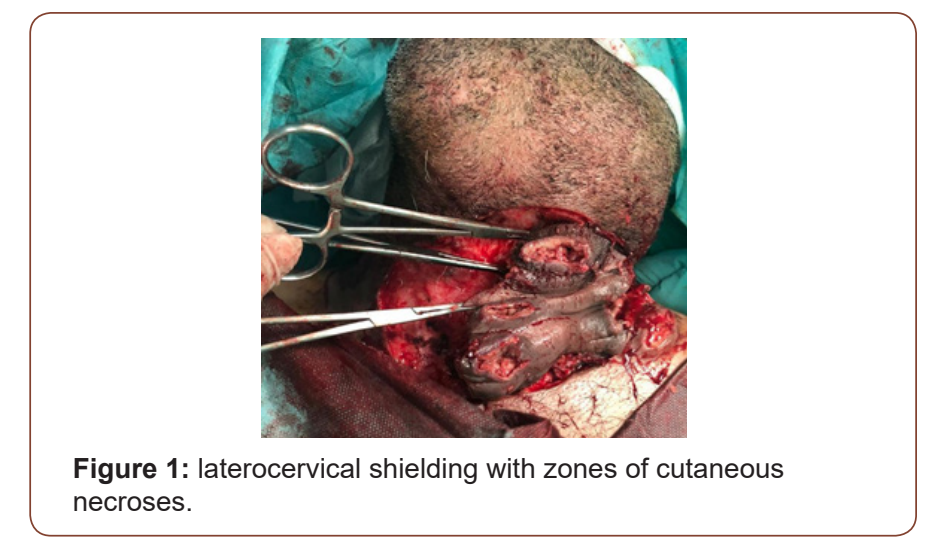

A cervicothoracic CT scan confirmed the diagnosis of cervical cellulitis, with areas of cutaneous necrosis without into thoracic extension. under general anaesthesia we performed 
a surgical removal with excision of the necrotic tissue (Figure 2). histopathological examination revealed a cutaneous non hodgkinien B cell lymphomas. After an extension check-up, the patient was given a chemotherapy (CHOP protocol) combined with radiotherapy at a dose of $55 \mathrm{~Gy}$.

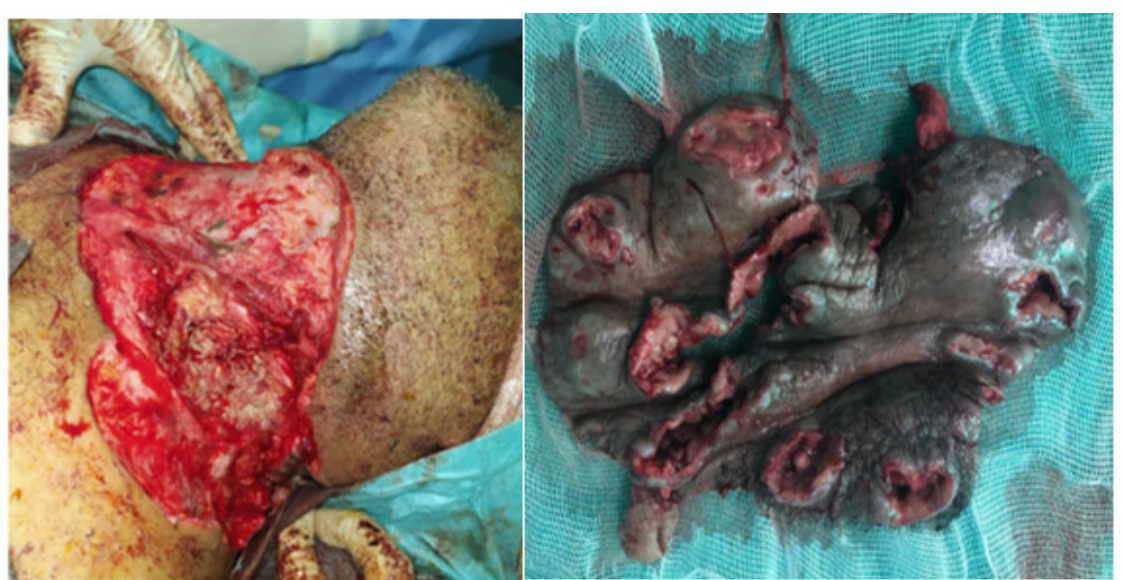

Figure 2: Aponevrotomy with draining and excision of the necrotic tissue.

\section{Discussion}

The location of LMNHs in the cervicofacial soft tissues is exceptional due to the rarity of the tissue lymphoide at this location. LMNH can be primitive, or most often secondary to the extension of a lymphoma of the lymph nodes or extranodal lymphoma, particularly of the parotid gland, which is rich in elements lymphoides [1]. Classically, cutaneous lymphomas occur under the following conditions the shape of papules, plate nodules, flaccid tumours, or even panniculitis [3]. The most deceptive aspect is that of cellulite in the soft tissues, especially in the area of cervicofacial. It is characterized by a rapid aggravation and the absence of clinical improvement despite treatment. well-conducted antibiotic.

Positive diagnosis of the primary skin location of the lymphoma is difficult to diagnose by imaging despite its progress. Other factors can lead to misdiagnosis, in particular the introduction of corticosteroid therapy which modifies the architecture of the tumour cells and makes it difficult to read the sections [2]. cutaneous lymphomas have the following characteristics and its own classification, that of the EORTC. It is based on clinical and morphological elements, immunological and evolutive. The interest of this classification is therapeutic. In fact, chemotherapy, which is the basis of the treatment, will be adapted to the aggressivity of the lymphoma [1-2].

The differential diagnosis includes mainly pseudolymphomas and primary cutaneous T-cell lymphomas (PCTCL), as well systemic B-cell lymphomas with cutaneous involvement [4]. The available therapeutic modalities are varied and include surgical excision, radiotherapy, corticosteroids, antibiotics, monoclonal antibodies and interferon, as well as monotherapy or multidrug therapy regimens. Treatment should be appropriate to the risk and chosen according to the type of lymphoma and clinical stage, as well as the characteristics of the patient and the number, type, extent, location and distribution of skin lesions [5].

\section{Conclusion}

this case is special because cellulite was a manifestation of cutaneous lymphoma which was confirmed on histopathological examination.

\section{Acknowledgement}

None.

\section{Conflict of Interest}

No Conflict of Interest.

\section{References}

1. Crickx B, Sigal-Grinberg M (2002) Lymphomes cutane's. Ann Dermatol Venereol 129: S153-155.

2. Burg G, Kaudewitz $P$ (1990) Where are we today in the diagnosis of cutaneous lymphoma? Curr Probl Dermatol 19: 90-104.

3. Querfeld C, Guitart J, Kuzel TM, Rosen ST (2003) Primary cutaneous lymphomas: a review with current treatment options. Blood Rev 17: 131-142.

4. Sokol L, Naghashpour M, Glass LF (2012) Primary cutaneous B-cell lymphomas: recent advances in diagnosis and management. Cancer Control 19: 236-244.

5. Burg G, Kempf W, Cozzio A, Feit J, Willemze R, et al. (2005) WHO/EORTC classification of cutaneous lymphomas 2005: histological and molecular aspects. J Cutan Pathol 32(10): 647-674. 